\title{
TOWARDS INTEGRATED OPTICAL GYROS USING BRILLOUIN LASERS
}

\author{
K. Vahala, Y. H. Lai, M. G. Suh, J. Li, K. Y. Yang \\ Watson Laboratory of Applied Physics, California Institute of Technology \\ Pasadena, California USA
}

\begin{abstract}
Ultra-low-loss micro-scale optical resonators have advanced to a point where $\mathrm{Q}$ factors of nearly 1 billion (monolithic devices $[1,2]$ ) and 100 billion (discrete devices [3]) are possible. These resonators have many applications including use for highcoherence compact lasers. Counter-propagating laser waves in these resonators can potentially enable the transfer of ring laser gyroscope technology to a semiconductor chip-based platform. Here, a monolithic optical gyroscope is described that uses counter-propagating Brillouin lasers to measure rotation through a Sagnac-induced frequency shift. Demonstration of rotation measurement below the Earth rotation rate is presented. Prospects for improving the device performance are described.
\end{abstract}

\section{INTRODUCTION}

Optical gyroscopes (ring-laser gyroscopes [4] and fiber-optic gyroscopes [5]) provide high-performance rotation sensors. However, MEMS-based rotation sensors, on account of their compact semiconductor-chip-based form factor and low cost, dominate consumer applications [6]. Nonetheless, the advent of monolithic optical resonators [7] has generated interest in chipbased analogs to the ring laser or fiber-optic gyroscope. Being lightweight with no moving parts, such micro-optical gyros [8] would be highly insensitive to shock and vibration. Moreover, their reliance on the Sagnac effect could potentially allow them to outperform MEMS devices. To date, however, the performance of micro-optical devices has severely lagged behind MEMS devices on account of difficult-to-achieve requirements for low-loss chipbased waveguides and high Q-factor optical resonators. Recent reports of micro-optical gyroscopes suggest that this landscape is beginning to change.

Both crystalline-based and silica-based optical rotation measurements have recently been demonstrated with performance that greatly exceeds prior micro-optical-gyro efforts $[9,10]$. These devices use advanced ultra-high-Q (UHQ) optical resonators [1-3]. In the resonators, light propagates in a circular, "whisperinggallery' optical path. For example, a recent monolithic UHQ silicaon-silicon ridge resonator is described in figure 1 [2]. Figure 1A shows a scanning electron micrograph of the resonator. At the far right is a silicon nitride waveguide (false color red) that couples light into the resonator near a region circumscribed with a dashed box in the image. As illustrated, light couples into a counterclockwise path shown by the yellow arrow. In a gyro measurement, Sagnac-induced changes in the resonant frequencies of clockwise and counter-clockwise optical modes are measured to detect rotation. The silicon nitride waveguide in the image can also function as an optical bus to convey the light to other devices on the chip for processing. In figure $1 \mathrm{~B}$ a concept rendering of a dual optical gyroscope is presented that would use two of the ridge resonator devices for redundant measurement. Other photonic components and electronics are shown on the chip. These can include III-V detectors and lasers that are die-attached at the wafer scale $[11,12]$.

In this presentation, Brillouin laser physics is first reviewed and then Brillouin gyro measurements are described. Finally, ways to improve the gyro performance are discussed.

\section{BRILLOUIN LASER GYROSCOPES}

Stimulated Brillouin scattering (SBS) has been intensely studied in silica optical fiber [13]. It results from the interaction of an optical pump wave with microwave-rate phonons. The application of this process for rotation sensing was also demonstrated using optical fibers in the 1990s [14].

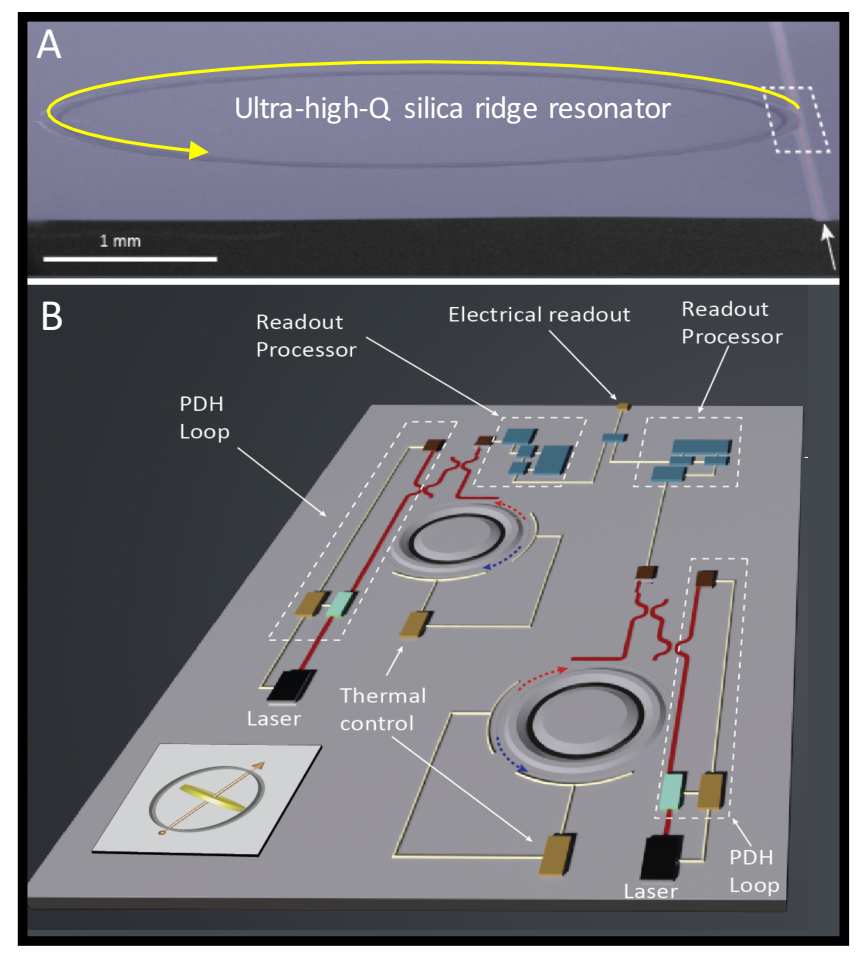

Figure 1: A. SEM image of an ultra-high-Q ridge resonator with a silicon nitride waveguide (false red color). White arrow shows the direction of light coupling and yellow arrow shows circulation within the resonator. Dashed white box is the waveguide-resonator coupling region. This material originally appeared in ref. [2]. $\boldsymbol{B}$. Concept rendering of a dual-gyro chip. Component color coding as follows: silicon nitride waveguides (red lines), electrical traces (silver lines), laser sources (black), detectors (brown), modulators (turquoise), loop-control electronics (gold), and readout processing electronics (blue).

To initiate Brillouin laser action, a resonator mode is optically pumped as shown in the figure $2 \mathrm{~A}$ (blue pump line). The ultrahigh-Q of the resonator leads to a strong build-up of the circulating intensity. $10 \mathrm{~s}$ of $\mathrm{MW} / \mathrm{cm}^{2}$ of circulating intensity is possible with only $1 \mathrm{~mW}$ of coupled optical pump power. This high-intensity field couples to phonons in the silica glass and, subject to a phase matching condition, will scatter off the phonons into other optical modes. In the whispering-gallery resonator, the scattering will efficiently couple the clockwise pump in figure $2 \mathrm{~A}$ into a counterclockwise "Stokes" resonant mode provided that the frequency of the Stokes mode is spectrally shifted relative to the pumping 
frequency by the Brillouin shift frequency $\left(\Omega_{\text {Brillouin }}\right.$ in figure $\left.2 \mathrm{~A}\right)$. For pump wavelengths in the telecom C-band (1.55 microns) this frequency shift is approximately $10.8 \mathrm{GHz}$ [13]. Moreover, in the high-Q resonator environment, the Brillouin coupling causes optical amplification of the Stokes wave. Upon sufficient optical pumping, this amplification overcomes the resonator loss and the Stokes wave will achieve laser threshold. Continued pumping above threshold causes a dramatic line narrowing of the Stokes laser field. In silica devices, this line narrowing achieves sub-Hertz fundamental linewidths [15]. Curiously, the Stokes laser wave can ultimately function as a pumping wave for a second Stokes wave, thereby setting up cascaded laser action in the resonator [15]. A measurement of the optical cascade process out to $9^{\text {th }}$ order is shown in figure 2B [15]. As shown in figure 2A each Stokes wave is shifted to lower frequencies and also switches the direction of propagation relative to its pumping wave. This natural switching of propagation direction sets up an ideal situation for measurement of rotations. Specifically, adjacent Stokes laser lines will experience opposing Sagnac-induced frequency shifts. To measure rotations, a neighboring pair of laser fields is photo-detected and the beat frequency is measured.

The sensitivity of this method of rotation measurement depends upon the fundamental linewidth of the beat note, which, as noted above, is sub-Hertz [15]. Additionally, because the counterpropagating Brillouin lasers are co-lasing within the same cavity, the technical noise contributions to their respective linewidths are largely common-mode-noise. As a result, heterodyne detection of the Brillouin laser waves produces a sub-Hertz beat frequency [16].
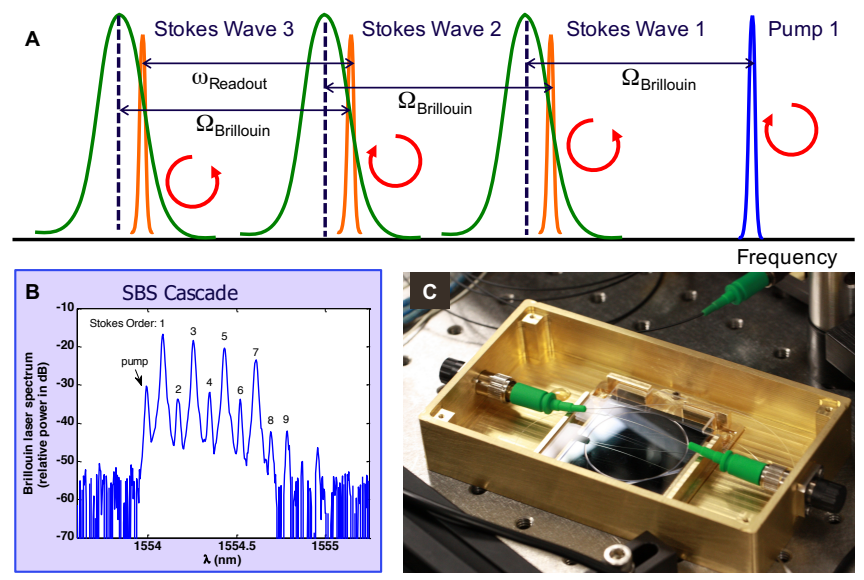

Figure 2: A. Principle of operation of gyroscope based on counterpropagating Brillouin laser oscillation. Stokes laser waves are shown in orange. Neighboring Stokes waves propagate in opposite directions and therefore experience opposing Sagnac frequency

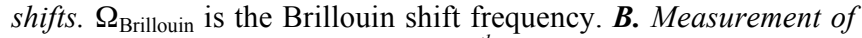
SBS cascaded laser action out to $9^{\text {th }}$ order. The odd orders are prominent in the measurement because the light output was collected in a direction opposite to the pumping direction. $\boldsymbol{C}$. Photograph of a disk resonator gyro packaged in a brass box with fiber connectors.

\section{ROTATION MEASUREMENT}

The current work uses a silicon-chip-based ultra-high-Q disk resonator [1] to generate counter-propagating Brillouin lasers for rotation measurement [9]. A photograph of a resonator packaged into a small box for rotation measurement is shown in figure $2 \mathrm{C}$. Light is coupled into and out from the box by fiber connectors.
When the resonator is rotated about an axis perpendicular to the plane of the disk, the counter-propagating Brillouin lasers experience opposing Sagnac frequency shifts. The impact of clockwise and counter-clockwise rotations on SBS $2^{\text {nd }}$ Stokes and SBS $3^{\text {rd }}$ Stokes wave is illustrated in figure $3 \mathrm{~A}$ and $3 \mathrm{~B}$. The measured beat frequency of these two waves (with a constant microwave-rate offset removed) is displayed in figure $3 \mathrm{C}$ when the resonator is subjected to a sinusoidal rotation. It is interesting to note that a 90-degree relative phase shift is observable between the rotation angle (blue) and Sagnac frequency shift (red) as is expected since the frequency shift is proportional to an angular rotation rate. Initial work on this device was able to measure rotations rates as low as 22 degrees/hour [9].

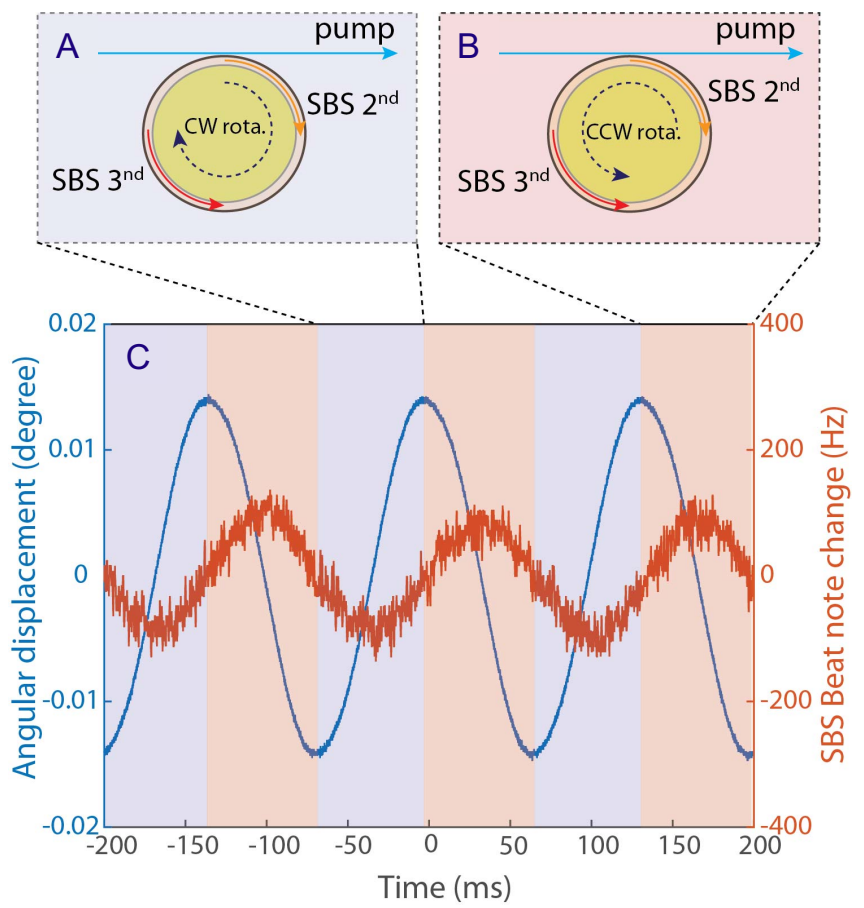

Figure 3: A. Rendering showing counter-propagating $2^{\text {nd }}$ and $3^{\text {rd }}$ Stokes waves in a whispering gallery resonator that is subject to a clockwise rotation. In this arrangement, the $2^{\text {nd }}$ Stokes wave decreases in frequency and the $3^{\text {rd }}$ Stokes wave increases in frequency, thereby causing a net decrease in their beat frequency subject to the Sagnac effect. B. Similar to panel A except resonator is now rotated in the counter-clockwise direction so that the beat frequency is increasing. C. Sinusoidal rotation measurement. Blue curve is the rotation angle and red is the measured Sagnac frequency shift generated by mixing Stokes 2 and Stokes 3 waves. This material originally appeared in ref. [9].

Improvements to the system have now boosted the bias stability to around 3.6 degrees/hour (see figure 4 ). In the newer design, the device is no longer being operated in cascaded fashion, but is using a dual-optical-pumping configuration. Allan deviation measurements of the beat frequency are presented in figure 4 (normalized to an equivalent rotation rate) for two different gyroscope resonator diameters. At short gate times, the system is limited by white frequency noise that is set by the fundamental linewidth of the co-lasing Stokes waves. At longer gate times, there is a drift component that is believed to be associated with thermal drift of the package. The overall improved performance of the larger diameter device is the result of its inherently smaller 
fundamental linewidth, its larger Sagnac coefficient, and its larger cavity mode volume which reduces drift. In these measurements there are no active thermal control circuits. Methods underway to address the drift component will be discussed as well as prospects for further reductions in the white frequency noise associated with the Stokes laser action.

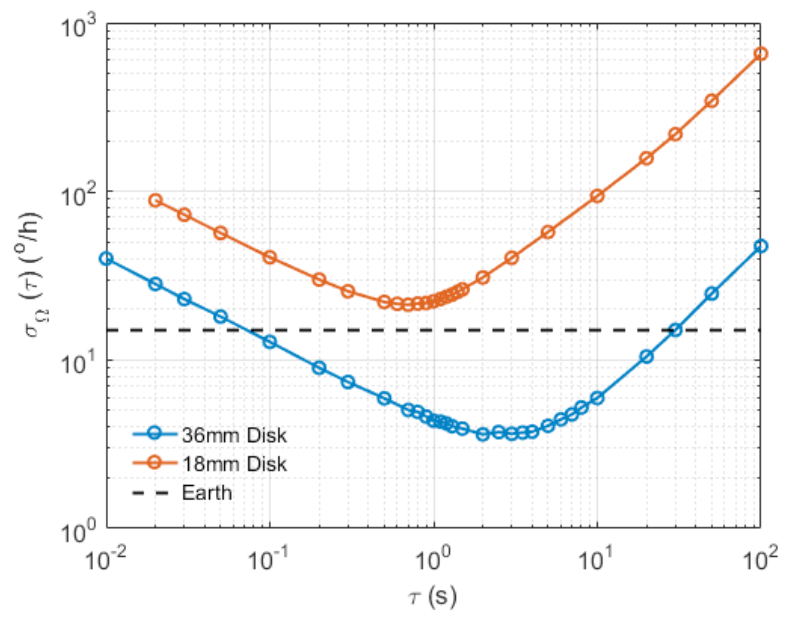

Figure 4: Allan deviation measurement performed on two disk resonator devices having different diameters (see legend).

\section{REFERENCES}

[1] Lee, H., Chen, T, Li, J., and Vahala, K, "Chemically etched ultra-high-Q wedge-resonator on a silicon chip," Nature Photonics, 6, 369 (2012).

[2] Yang, K. Y., Oh, D. Y., Lee, S. H., Yang, Q. F., Yi, X., Shen, B., Wang, H., and Vahala, K., "Bridging ultrahigh-Q devices and photonic circuits," Nature Photonics, doi:10.1038/s41566-018-0132-5 (2018).

[3] Grudinin, I. S., Ilchenko, V. S., and Maleki, L., "Ultrahigh optical $Q$ factors of crystalline resonators in the linear regime," Phys. Rev. A, 74, 063806 (2006).

[4] Chow, W. et al., "The ring laser gyro," Reviews of ModernPhysics 57, 61 (1985).

[5] Lefevre, H. C., "The fiber-optic gyroscope," (Artech house, 2014).

[6] Liu, K. et al., "The development of micro-gyroscope technology," Journal of Micromechanics and Microengineering 19, 113001 (2009).

[7] Vahala, K. “Optical Microcavities," Nature, 424, 839 (2003).

[8] Dell'Olio, F., Tatoli, T., Ciminelli, C., and Armenise, M., "Recent advances in miniaturized optical gyroscopes," Journal of the European Optical Society-Rapid publications 9 (2014).

[9] Li, J., Suh, M. G., and Vahala, K., "Microresonator Brillouin gyroscope," Optica 4, 346 (2017).

[10] Liang, W., Ilchenko, V. S., Savchenkov, A. A., Dale, E., Eliyahu, D., Matsko, A. B., and Lute, M., "Resonant microphotonic gyroscope," Optica 4, 114 (2017).

[11] Komljenovic, T. et al., "Heterogeneous silicon photonic integrated circuits," J. Light. Technol. 34, 20 (2016).

[12] Piels, M., Bauters, J. F., Davenport, M. L., Heck, M. J., and Bowers, J. E., "Low-loss silicon nitride AWG demultiplexer heterogeneously integrated with hybrid III-V/silicon photodetectors," J. Light. Technol. 32, 817 (2014).

[13] E. Ippen and R. Stolen, "Stimulated Brillouin scattering in optical fibers," Appl. Phys. Lett. 21, 539 (1972).
[14] F. Zarinetchi, S. Smith, and S. Ezekiel, "Stimulated Brillouin fiber-optic laser gyroscope,” Optics Letters 16, 229 (1991).

[15] Li, J., Lee, H., Chen, T., and Vahala, K., "Characterization of a high coherence, Brillouin microcavity laser on silicon," Optics Express, 20, 20170 (2012).

[16] Li, J., Lee, H., Vahala, K., "Microwave synthesizer using an on-chip Brillouin oscillator," Nature Communications 4, 2097 (2013).

\section{CONTACT}

*Kerry Vahala, vahala@,caltech.edu

\section{ACKNOWLEDGEMENT}

The authors gratefully acknowledge the Defense Advanced Research Projects Agency (DARPA) under the PRIGM:AIMS program (grant no. N66001-16-1-4046) and the Kavli Nanoscience Institute. 